\title{
The Progress and Future of Near-Field Radiative Heat Transfer
}

\author{
Zhiheng Zheng, ${ }^{1,2}$ Wenwen Liang, ${ }^{1}$ Shaolan Zhong ${ }^{1}$ and Wei $\mathrm{Yu}^{1,2, *}$
}

Received date: 15 April 2021; Accepted date: 5 May 2021

Article type: Editorial article.

As a fundamental method of heat transfer, thermal radiation spontaneously occurs for any material with nonzero kelvin temperature. The radiative heat transfer corresponds to the energy transfer between objects through thermal radiation. So far, the radiative heat transfer is mainly divided into two categories, which are determined by the relative size of the characteristic geometric dimension of objects and the dominant wavelength of thermal radiation determined from Wien's displacement law. For example, when the dominant wavelength of thermal radiation is much smaller than the distance between the heat source and heat sink, the radiative heat transfer is called far-field radiative heat transfer (FFRHT). The corresponding radiative heat flux is limited by the wellknown Stefan-Boltzmann law. ${ }^{[1]}$ This is because that the radiative heat transfer channel (i.e., wave vector $k$ ) is limited in the propagating modes $(k<\omega / c)$, where $\omega$ is the angular frequency and $c$ is the light speed in the vacuum. ${ }^{[2,3]}$ Instead, when the dominant wavelength of thermal radiation is comparable to or even larger than the distance between the heat source and heat sink, the radiative heat transfer is called near-field radiative heat transfer (NFRHT). The corresponding radiative heat flux can break through the well-known StefanBoltzmann law and exceed the radiative heat flux predicted by the well-known Stefan-Boltzmann law even several orders of magnitude. ${ }^{[2-6]}$ This is because that the radiative heat transfer channel $(k)$ can extend from the propagating modes to the evanescent modes $(k>\omega / c){ }^{[2,3]}$ Meanwhile, the radiative heat flux can be further enhanced due to near-field effects, such as excitation of surface waves including surface phonon polaritons (SPhPs) and surface plasmon polaritons (SPPs), which can effectively enhance the transmission factor for evanescent modes. ${ }^{[4-6]}$ During the past few decades, this feature

${ }^{1}$ School of Environmental and Materials Engineering, College of

Engineering, Shanghai Polytechnic University, Shanghai 201209,

China.

${ }^{2}$ Shanghai Engineering Research Center of Advanced Thermal

Functional Materials, Shanghai Polytechnic University, Shanghai

201209, China.

*E-mail: yuwei@sspu.edu.cn (W. Yu) of NFRHT has attracted a lot of attention, including theoretical and experimental research. Especially in the past decade, the theoretical and experimental research of NFRHT has made continuous breakthroughs, and the theoretical research of NFRHT has been verified by much experimental research, attracting more and more scholars to join the research of this field. ${ }^{[2-8]}$ With the development of new materials and structures in NFRHT field, the physical mechanism and potential application of the NFRHT have been greatly enriched. Therefore, in this editorial article, we would like to give a brief overview of NFRHT.

Conventional isotropic polar materials, semiconductor materials, and metal materials may be welcomed and studied by researchers earlier. The SPhPs supported by polar materials and the SPPs supported by semiconductor materials and metal materials can result in the transmission factor keeping high value to large wave vector $k$ for evanescent waves in a narrow angular frequency band. ${ }^{[2-6]}$ This can greatly enhance the radiative heat flux and make it monochromatic (i.e., sharp peak in the spectral radiative heat flux,,$^{[2-6]}$ and thus is very important in enhancing and regulating NFRHT. However, these materials are usually nonmagnetic, and thus the SPhPs and SPPs are mainly excited in the $p$-polarization, limiting their way to enhance and regulate the NFRHT. Due to supporting the surface waves in the $s$-polarization, metamaterials have been involved to further enhance and regulate NFRHT. ${ }^{[9,10]}$ Accordingly, it can be seen that the NFRHT can be significantly affected by the excitation of surface waves. Consequently, due to the adjustable SPPs from terahertz to mid-infrared, graphene exhibits great potential in the enhancement and regulation of NFRHT, which has been confirmed by many subsequent studies. ${ }^{[1-13]}$ Graphene is a representative two-dimensional material, and its great progress in the field of NFRHT has also inspired scholars to explore the effect of other two-dimensional materials such as molybdenum disulfide $\left(\mathrm{MoS}_{2}\right)$ materials and black phosphorus sheets on NFRHT. ${ }^{[14-16]}$ In addition to extending nonmagnetic materials to metamaterials, researchers also extend isotropic materials to various anisotropic materials, along with different physical mechanisms which can 
significantly affect NFRHT. As a typical anisotropic material with a simple dielectric function, the uniaxial hyperbolic material has been widely studied. ${ }^{[17-19]}$ Hyperbolic modes (HMs) supported by it and later further developed into hyperbolic surface phonon polaritons (HSPPs) and hyperbolic volume phonon polaritons (HVPPs), provide new ways to enhance and regulate NFRHT. ${ }^{\left[{ }^{[18,19]} \text { Subsequently, anisotropic }\right.}$ magneto-dielectric hyperbolic metamaterials supporting HMs both in the $s$ and $p$ polarizations, and more general anisotropic materials further enrich the physical mechanisms of enhancing and regulating the NFRHT. ${ }^{[20]}$ Recently, based on anisotropic materials, magneto-optical materials whose dielectric function can be regulated by an external magnetic field have been proposed to enhance and regulate NFRHT, exhibiting high adjustability and nonreciprocity for NFRHT. ${ }^{[21-23]}$ Another important advance in the materials for NFRHT is the use of phase change materials. Although there may be not many new mechanisms significantly affecting NFRHT, these materials provide many new potential ways for the applications of NFRHT, ${ }^{[13,24-27]}$ also attracting the attention of many scholars. From the above discussion, the introduction of new materials into NFRHT is likely to bring new physical mechanisms to enhance and regulate NFRHT, or new potential ways for the applications of NFRHT, along with challenges and opportunities. Therefore, we should encourage more explorations of how new materials affect NFRHT to reveal the possible new physical mechanisms, providing new ways for the enhancement and regulation of NFRHT, or opening up some new potential applications of NFRHT.

On the other hand, from the perspective of the research structure of NFRHT, the two-body system (including two planar objects, a sphere and a planar substrate, two spheres) is studied by researchers earlier. ${ }^{[28]}$ The SPhPs and SPPs may be the main physical mechanisms corresponding to the two-body system. Then with uniaxial hyperbolic materials NFRHT, the HMs become another main physical mechanism for the twobody system. ${ }^{[17-21]}$ The uniaxial hyperbolic materials can be natural or equivalent based on the effective medium theory. With the development of the calculation methods of the NFRHT, the two-body system has developed to include gratings and other microstructures, ${ }^{[29-34]}$ and the corresponding physical mechanism has become more abundant. For example, even for conventional isotropic materials, the guided modes (GMs), HMs, spoof surface plasmon polartions (SSPPs), magnetic polaritons (MPs), and SPPs all can be the important physical mechanisms significantly affecting the NFRHT between two gratings. ${ }^{[29-33]}$ Therefore, when materials are anisotropic or other unconventional materials, it can be inferred that there would be more possible new physical mechanisms significantly affecting NFRHT to be developed. Another important development for the structure of NFRHT is extending the two-body system to the many-body system. ${ }^{[35-37]}$ Due to the interaction between the many bodies, the hybridization or coupling of the physical modes in the system is improved and the regulation of NFRHT including enhancement and suppression is more flexible. By cooperation with conventional and specific materials, such as uniaxial hyperbolic materials, magneto-optical materials, Weyl semimetals, phase change materials, etc., the many-body system can exhibit the waveguide effect, photon thermal hall effect, superdiffusive and ballistic heat transport, nonreciprocity, anomalous photon thermal hall effect, etc. ${ }^{[22,38-42]}$ Meanwhile, the many-body system can greatly enrich the potential application of NFRHT, including the thermal transistor, heat engine, thermal logic gates, nonreciprocal thermal diode, heat flux switch, heat pump, etc. ${ }^{[22,25,27,42-45]}$ Therefore, compared with the two-body system, the many-body system may exhibit more anomalous NFRHT phenomena and potential applications. However, the physical mechanisms behind these phenomena need to be further explored.

In summary, NFRHT has been greatly developed by the efforts of scholars in the past decades. However, it still has a lot to be improved. Although experimental research has made many breakthroughs in recent years, it is still backward compared with theoretical research. The experimental research mainly focuses on conventional materials and simple structures, and that for complex structures such as many-body system needs to be further developed. Secondly, it is still challenging to introduce new materials into NFRHT and explore the possible new physical mechanism, providing new ways for the enhancement and regulation of NFRHT, or opening up some new potential applications of NFRHT, but it is worth encouraging. Finally, we should actively develop more unique structures cooperating with conventional or specific materials in NFRHT, analyze the possible new physical mechanism, and explore the new potential applications. We believe that through the unremitting efforts of scholars, NFRHT would play an important role in the applications of thermal management, energy conversion system, scanning thermal microscope, and so on.

\section{Acknowledgements}

The authors express sincere thanks to the financial support from National Natural Science Foundation of China (51806134, 51876112).

\section{References}

[1] F. P. Incropera, D. P. DeWitt, Fundamentals of Heat and Mass Transfer. New York: John Wiley \& Sons, ed. Fifth Edition, 2002. [2] J. B. Pendry, J. Phys. Condens. Matter, 1999, 11, 6621-6634. [3] A. I. Volokitin, B. N. J. Persson, Rev. Mod. Phys., 2007, 79, 1291-1329.

[4] S. A. Biehs, E. Rousseau, J. J. Greffet, Phys. Rev. Lett., 2010, $\mathbf{1 0 5}, 234301$.

[5] J. P. Mulet, K. Joulain, R. Carminati, J. J. Greffet, Microscale Thermophys. Eng., 2002, 6, 209-222.

[6] S. Basu, B. J. Lee, Z. M. Zhang, J. Heat Transf., 2010, 132, 023302.

[7] E. Rousseau, A. Siria, G. Jourdan, S. Volz, F. Comin, J. Chevrier, J. J. Greffet, Nat. Photonics, 2009, 3, 514-517. 
[8] B. Song, D. Thompson, A. Fiorino, Y. Ganjeh, P. Reddy, E. Meyhofer, Nat. Nanotechnol., 2016, 11, 509-515.

[9] K. Joulain, J. Drevillon, P. Ben-Abdallah, Phys. Rev. B, 2010, 81, 165119.

[10] Z. Zheng, Y. Xuan, Int. J. Heat Mass Transf., 2011, 54, $1101-$ 1110 .

[11] O. Ilic, M. Jablan, J. D. Joannopoulos, I. Celanovic, H. Buljan, M. Soljačić, Phys. Rev. B, 2012, 85, 155422.

[12] R. Messina, P. Ben-Abdallah, Sci. Rep., 2013, 3, 1383.

[13] Z. Zheng, X. Liu, A. Wang, Y. Xuan, Int. J. Heat Mass Transf., 2017, 109, 63-72.

[14] J. Peng, G. Zhang, B. Li, Appl. Phys. Lett., 2015, 107, 133108 .

[15] J. Shen, S. Guo, X. Liu, B. Liu, W. Wu, H. He, Appl. Therm. Eng., 2018, 144, 403-410.

[16] Y. Zhang, H. Yi, H. Tan, ACS Photonics, 2018, 5, 3739-3747.

[17] S. A. Biehs, M. Tschikin, P. Ben-Abdallah, Phys. Rev. Lett., 2012, 109, 104301.

[18] X. Liu, Y. Xuan, Sci. China Technol. Sci., 2016, 59, 16801686.

[19] X. Wu, C. Fu, J. Quant. Spectroscopy. Radiat. Transf., 2021, 258, 107337.

[20] J. Song, Q. Cheng, Phys. Rev. B, 2016, 94, 125419.

[21] E. Moncada-Villa, V. Fernández-Hurtado, F. J. García-Vidal, A. García-Martín, J. C. Cuevas, Phys. Rev. B, 2015, 92, 125418. [22] A. Ott, R. Messina, P. Ben-Abdallah, S. A. Biehs, Appl. Phys. Lett., 2019, 114, 163105.

[23] M. He, H. Qi, Y. Ren, Y. Zhao, M. Antezza, Int. J. Heat Mass Transf., 2020, 150, 119305.

[24] C. R. Otey, W. T. Lau, S. Fan, Phys. Rev. Lett., 2010, 104, 154301 .

[25] P. Ben-Abdallah, S. A. Biehs, Phys. Rev. Lett., 2014, 112, 044301.

[26] V. Kubytskyi, S. A. Biehs, P. Ben-Abdallah, Phys. Rev. Lett., 2014, 113, 074301.

[27] P. Ben-Abdallah, S. A. Biehs, Phys. Rev. B, 2016, 94, 241401(R).

[28] A. I. Volokitin, B. N. J. Persson, Phys. Rev. B, 2001, 63, 205404.

[29] R. Guérout, J. Lussange, F. S. S. Rosa, J. P. Hugonin, D. A. R. Dalvit, J. J. Greffet, A. Lambrecht, S. Reynaud, Phys. Rev. B, 2012, 85, 180301(R).

[30] X. Liu, B. Zhao, Z. M. Zhang, Phys. Rev. A, 2015, 91, 062510 .

[31] J. Dai, S. A. Dyakov, S. I. Bozhevolnyi, M. Yan, Phys. Rev. $B, 2016,94,125431$.

[32] Y. Yang, L. Wang, Phys. Rev. Lett., 2016, 117, 044301.

[33] V. Fernández-Hurtado, F. J. García-Vidal, S. Fan, J. C. Cuevas, Phys. Rev. Lett., 2017, 118, 203901.

[34] A. Didari, M. Pinar Mengüç, J. Quant. Spectrosc. Radiat. Transf., 2017, 197, 95-105.

[35] P. Ben-Abdallah, S. A. Biehs, Phys. Rev. Lett., 2011, 107, 114301.

[36] Z. Zheng, Y. Xuan, Nanoscale Microscale Thermophys. Eng., 2011, 15, 237-251.
[37] R. Messina, M. Antezza, P. Ben-Abdallah, Phys. Rev. Lett., 2012, 109, 244302.

[38] R. Messina, P. Ben-Abdallah, B. Guizal, M. Antezza, S. A. Biehs, Phys. Rev. B, 2016, 94, 104301.

[39] P. Ben-Abdallah, Phys. Rev. Lett., 2016, 116, 084301.

[40] I. Latella, S. A. Biehs, R. Messina, A. W. Rodriguez, P. BenAbdallah, Phys. Rev. B, 2018, 97, 035423.

[41] A. Ott, S. A. Biehs, P. Ben-Abdallah, Phys. Rev. B, 2020, 101, 241411(R).

[42] J. Song, Q. Cheng, B. Zhang, L. Lu, X. Zhou, Z. Luo, R. Hu, Rep. Prog. Phys., 2021, 84, 036501.

[43] I. Latella, A. Pérez-Madrid, J. M. Rubi, S. A. Biehs, P. BenAbdallah, Phys. Rev. Appl., 2015, 4, 011001.

[44] M. He, H. Qi, Y. Li, Y. Ren, W. Cai, L. Ruan, Int. J. Heat Mass Transf., 2019, 137, 12-19.

[45] R. Messina, P. Ben-Abdallah, Phys. Rev. B, 2020, 101, 165435 .

Publisher's Note: Engineered Science Publisher remains neutral with regard to jurisdictional claims in published maps and institutional affiliations 